\title{
PERCEPCIÓN DEL RIESGO VOLCÁNICO Y CONOCIMIENTO DE LOS PLANES DE EMERGENCIA EN LOS ALREDEDORES DEL VOLCÁN POÁS, COSTA RICA
}

\author{
VOLCANIC RISK PERCEPTION AND EMERGENCY PLANS AWARENESS OF THE \\ POPULATION IN THE NEIGHBORHOOD OF VOLCANO POAS, COSTA RICA
}

\author{
Yessika Blunda ${ }^{1,2}$ \\ ${ }^{1}$ Escuela Centroamericana de Geología, Universidad de Costa Rica \\ ${ }^{2}$ Dirección actual: 12910 Westleigh Dr, Houston, TX 77077 \\ yblunda@yahoo.com
}

(Recibido: 14/08/2009; aceptado: 08/11/2010)

\begin{abstract}
The present article describes the volcanic risk and emergency plans awareness of the population surrounding the Poás volcano. A sample of the neighboring communities was surveyed in regards to their perception of the likelihood of a volcanic eruption and its severity. This survey showed that the risk of living in the neighborhood of the volcano is perceived as extremely low or inexistent. The neighboring population feels much attached to the area due to the economic advantages of its touristic attractiveness and fertile soils for agriculture. Only the individuals in scholar ages showed awareness of the existing emergency plans. This, in contrast with older individuals surveyed who just had a vague notion of where to evacuate. The root cause of this lack of volcanic risk awareness is insufficient promotion of emergency plans, and also the lack of a formal mechanism to periodically update the plan. This work sets the basis for an improved emergency plan to mitigate the consequences of a volcanic crisis, incorporating current demographic data from the latest census, a more accurate description of evacuation routes, and adding aspects ignored in previous versions related to health. It also describes effective means to divulgate the emergency plans, other than instruction in a classroom, for example, brochures handed to visitors and the neighboring community in general. This article was extracted from the author's thesis, which describes the state or activity of Poás Volcano in 2006, with geochemical and volcanic seismicity data.
\end{abstract}

Keywords: Costa Rica, Poás volcano, emergency plan, volcanic risk, community.

RESUMEN: El presente artículo describe la percepción de la población que rodea al volcán Poás en relación al riesgo volcánico y los planes de emergencia existentes. Se realizó una encuesta a una muestra de las comunidades vecinas respecto a su apreciación de la posibilidad de una erupción volcánica y su severidad. Esta encuesta mostró que la población percibe el riesgo de vivir en el área extremamente bajo o no riesgoso. Las poblaciones aledañas se encuentran muy arraigadas a la zona debido a su atractivo turístico y suelos fértiles para la agricultura. Sólo los individuos en edad 
escolar mostraron conocimiento básico de los planes de emergencia en contraste con los pobladores mayores, quienes sólo mostraron noción de hacia dónde evacuar. Las causas principales de esta falta de conciencia del riesgo volcánico es insuficiente divulgación de los planes de emergencia y falta de un mecanismo formal para actualizarlo periódicamente. Este trabajo sienta las bases para un mejor plan de emergencias con el fin de mitigar las consecuencias de una crisis volcánica. La propuesta incorpora datos demográficos actuales, de acuerdo con el censo más reciente, una descripción más adecuada de las rutas de evacuación, e incluyendo aspectos relacionados con la salud, que fueron ignorados en versiones anteriores. Este plan también describe otros métodos de divulgación del plan de emergencia, como por ejemplo, folletos que se entreguen a los visitantes del volcán Poás y la comunidad vecina, en general. Este artículo se extrajo de la tesis de maestría de la autora, la cual describe la actividad del volcán Poás para el año 2006, a través de mediciones de sismicidad volcánica y datos geoquímicos.

Palabras claves: Costa Rica, volcán Poás, plan de emergencias, riesgo volcánico, comunidad.

\section{INTRODUCCIÓN}

La Cordillera Volcánica Central está formada por cinco complejos estratovolcánicos principales (Platanar, Poás, Barva, Irazú y Turrialba), alineados en dirección aproximada $\mathrm{N} 60^{\circ} \mathrm{W}$. Existen reportes de actividad en el volcán Poás desde el año 1828 hasta el presente, siendo las erupciones más mencionadas las de 1910 y 1953-1955. Desafortunadamente son varias las localidades y miles los pobladores que se pueden ver afectados durante una crisis volcánica en el Poás. Muchos de los habitantes de esta área no perciben al volcán como un riesgo para sus vidas y sus propiedades, sino más bien una fuente de ingresos debido al atractivo turístico del Parque Nacional. Además, los pobladores se encuentran muy arraigados a la zona ya que han habitado allí por varias generaciones. En Costa Rica existen una serie de planes de contingencia y evacuación para salvaguardar la integridad física de los habitantes de áreas aledañas y de sus actividades económicas, así como también daños al ambiente. Los más recientes fueron realizados en el año 1994. Sin embargo, doce años más tarde (2006), no se ha realizado ningún tipo de verificación o actualización de los mismos por lo cual estos no reflejan las condiciones actuales de cada localidad. Además, con frecuencia dichos planes son desconocidos o pobremente adoptados por la comunidad, por lo que su sola existencia no puede contrarrestar los efectos de una erupción. Este trabajo fue diseñado con el fin de dar a conocer cuál es la percepción del riesgo volcánico en los alrededores del Volcán Poás y presentar una propuesta de actualización y efectiva adopción del Plan de Emergencias.

\section{GENERALIDADES DEL VOLCÁN POÁS}

El volcán Poás se encuentra ubicado en las coordenadas $10^{\circ} 15^{\prime}$ latitud norte y $84^{\circ} 14^{\prime}$ longitud oeste, entre los volcanes Platanar y Barva, en la Cordillera Volcánica Central de Costa Rica (Fig. 1). Su altura es de 2708 m. s.n.m y representa un estratovolcán complejo de $300 \mathrm{~km}^{2}$ de área, de forma subcónica irregular, en donde se presentan depresiones limitadas por fallas, conos volcánicos y cráteres, que se distribuyen según patrones de la tectónica y que son el producto de la actividad más reciente (Alvarado, 2000).

El volcán Poás está compuesto por tres estructuras principales: el cráter principal, caracterizado por un domo lávico (laguna caliente) y dos terrazas en el sector este; el cono con la Laguna Botos o Fría y el antiguo cono Von Frantzius. Es posible distinguir cuatro unidades morfogenéticas dentro del cráter principal: la meseta oriental, la laguna cratérica, la cúpula de lava y la playa interna (Casertano et al., 1983). La laguna cratérica es el producto de la acumulación del agua de lluvia y de pequeños aportes acuíferos locales. En ciertos períodos del siglo pasado se ha secado temporalmente, como en el caso de las erupciones de 1953 y en 1964. Este volcán se caracteriza por una gran actividad fumarólica originada por la desgasificación de 


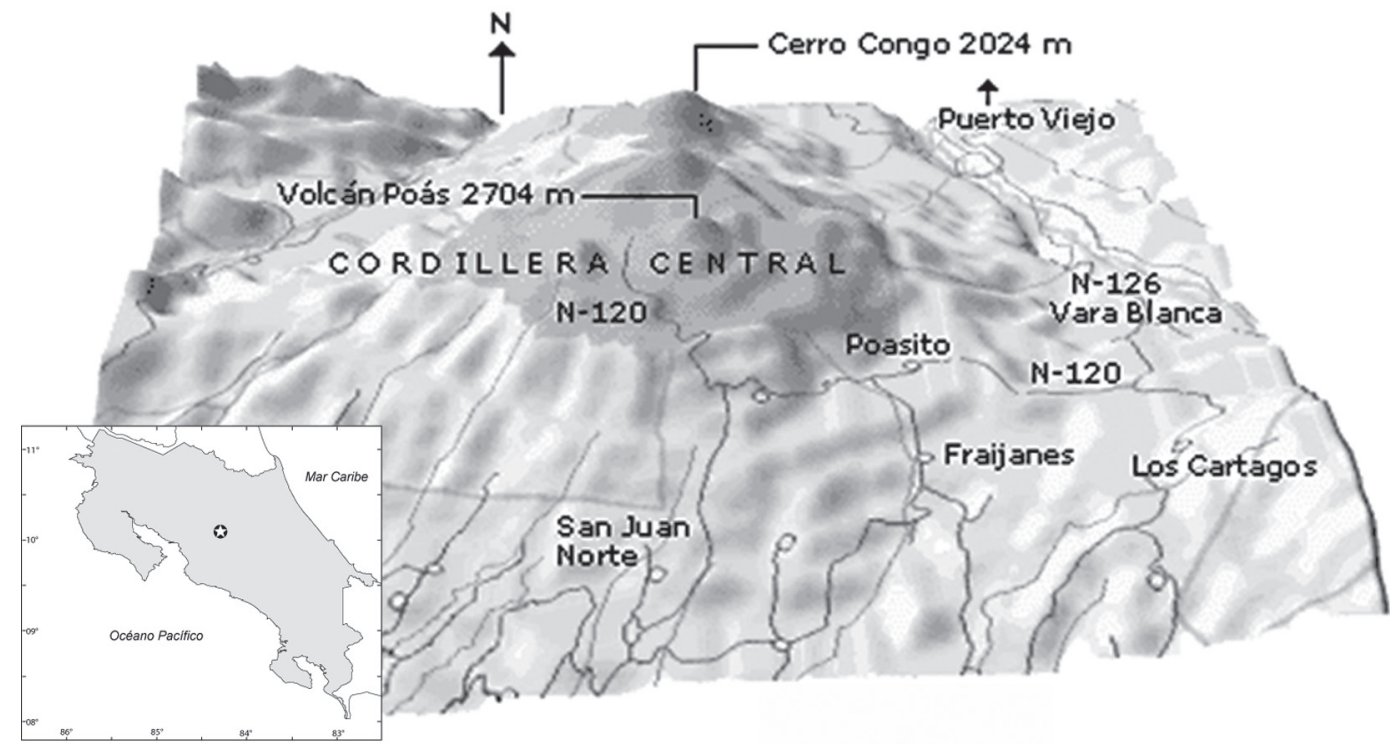

Fig. 1: Ubicación geográfica del volcán Poás.

cuerpos magmáticos superficiales. Estos gases están compuestos principalmente por $\mathrm{H}_{2} \mathrm{O}, \mathrm{H}_{2}, \mathrm{CO}_{2}$, $\mathrm{SO}_{2}, \mathrm{~S}, \mathrm{HCL}$ y HF. Se ha estimado que el volcán Poás emite columnas de gases que expulsan a la atmósfera varios cientos de toneladas diarias de $\mathrm{SO}_{2}$, las cuales dan el carácter ácido a las precipitaciones del lugar.

El Poás es un volcán activo y ha permanecido así desde tiempos pre-coloniales, con típicas erupciones seudo-geysiformes o freáticas de tamaño y frecuencias variadas, todas originadas en la laguna cratérica termo - mineral. Entre las erupciones más importantes se pueden destacar las de 1834 (erupciones de ceniza con fuertes detonaciones subterráneas), 1910 y las del período 1953 - 1955, de tipo freatomagmático y magmático (Casertano et al, 1983).

\section{RIESGO VOLCÁNICO}

La experiencia en emergencias volcánicas ha mostrado que es sumamente difícil mantener una percepción equilibrada del riesgo volcánico y que el nivel de conciencia depende fundamentalmente del tiempo transcurrido desde la última erupción devastadora en la misma región (UNESCO, 1987).
En el caso del Poás, una erupción devastadora como tal no ha ocurrido en tiempos históricos.

En vista de la importancia de los valores subjetivos en la percepción del riesgo, es aconsejable que cuando la seguridad y el bienestar de toda una comunidad están en peligro, la evaluación de la naturaleza y el grado de riesgo se hagan lo más objetivamente posible. Con el progreso de los conocimientos científicos y el mejoramiento de los sistemas de vigilancia volcánica, se debe esperar que evaluaciones fiables de los peligros y riesgos volcánicos se vuelvan progresivamente más disponibles para quienes toman decisiones (individuales y colectivas) durante emergencias volcánicas.

Desde la perspectiva del Riesgo, los estudios y análisis de vulnerabilidad, deben ser efectuados por el sistema local de emergencias, bajo la responsabilidad y coordinación de las municipalidades de las respectivas comunidades en riesgo. Se debe tener presente que los análisis y evaluaciones de amenaza y vulnerabilidad, permitirán la zonificación del riesgo volcánico, en sus distintos grados o niveles (UNESCO,1987).

En muchos países, cuando se declara estado de emergencia, la responsabilidad recae sobre un alto funcionario de la autoridad local, y no 
es extraño que éste tome una posición muy precavida en cuanto al riesgo que se pueda tolerar (UNESCO, 1987). El grado en que la seguridad de la población está bajo la responsabilidad de ese solo individuo o autoridad es otro de los factores que puede influir en la percepción del riesgo.

Un adecuado manejo de los factores de amenaza y vulnerabilidad asociados con el impacto de la actividad volcánica sobre su entorno constituye la base para una eficiente gestión del riesgo volcánico. Además de los factores de vulnerabilidad asociados a los fenómenos generados por la actividad volcánica en sí, se deben considerar otros aspectos que pueden a su vez aumentar o disminuir la vulnerabilidad de la comunidad a la amenaza de una erupción volcánica, tales como la organización social y gestión de la comunidad para hacer frente a una emergencia o desastre de origen volcánico. Por ejemplo:

. Factores culturales de la comunidad local

. Nivel de conocimiento de los riesgos a que está expuesta la comunidad

. Tipo y diseño de viviendas

. Densidad y distribución de la población servicios

. Nivel de equipamiento e infraestructura de

. Densidad de la red vial

. Diversidad de la economía local

. Nivel de gestión frente al riesgo volcánico

. Nivel de capacitación y preparación de los organismos e instituciones componentes del comité de emergencias local

\section{PERCEPCIÓN DEL RIESGO VOLCÁNICO EN EL VOLCÁN POÁS}

En el caso del volcán Poás, son varias las poblaciones que se pudieran ver afectadas en caso de ocurrir una erupción. La figura 2 muestra las localidades de Alajuela que podrían ser afectadas por lluvias de ceniza volcánica. Entre ellas se encuentran Carbonel, San Miguel, San Isidro de Poás, Poasito, San Pedro Poás, San Roque, San Rafael, Sabana Redonda y Fraijanes. Precisamente, en estas localidades fue realizada una encuesta a alrededor 200 pobladores de la zona a fin de conocer cuál es su percepción del riesgo volcánico y la existencia de planes de emergencia ante una erupción del volcán Poás (Anexo 1). La mayoría de los entrevistados se encontraban en edades comprendidas entre los 31 y 40 años.

Un $60 \%$ de la población entrevistada expresó que vive en el área porque su familia ha vivido allí generación tras generación y nunca han pensado en abandonar estas localidades. Con esto, fue posible observar el arraigo de la gente a un lugar específico sin importar la vulnerabilidad de la zona. Por otra parte un 33\% de la población expresó vivir allí por motivos laborales, ya que debido a la fertilidad de los suelos, han conseguido trabajo en la agricultura y se encuentran satisfechos en esas poblaciones ya que según comentan, en otros sitios no podrían conseguir el trabajo que ahora tienen. Estas personas no tienen la perspectiva del riesgo asociado con habitar la zona en cuestión, es decir, no se dan cuenta que en cualquier momento podrían perder sus cosechas debido a los efectos de la lluvia ácida o la caída de ceniza sobre los productos agrícolas. Seguidamente, se preguntó a los entrevistados si consideraban que vivían en una zona de peligro volcánico. El 58\% de la población contestó que no, mientras que un $42 \%$ contestó que sí. La mayoría de la población no acepta que vive en una zona de peligro volcánico. Sin embargo, aseguraron que algunos de sus vecinos que se encuentran a tan solo $5 \mathrm{~km}$ de distancia, sí viven en una zona de riesgo.

El $73 \%$ de la población encuestada estaría dispuesta a abandonar sus propiedades en caso de presentarse una erupción volcánica. Estas personas manifestaron que de ser necesario lo harían, ya que "la vida está por encima de las cosas materiales". Un $16 \%$ no se encuentra de acuerdo en dejar sus propiedades por miedo a que les roben, dañen sus viviendas o campos de cultivo, algunos delincuentes que pudieran llegar a la zona. Esto podría ser un factor negativo y de dificultad a la hora de la activación del plan de emergencias ya que estas personas no acatarían las órdenes de las autoridades y podrían ser causantes de problemas en las labores de evacuación y reubicación temporal en albergues.

Con respecto a las vías de evacuación, la población tiene una noción acerca de qué lugares debe dirigirse, según su instinto de supervivencia, 


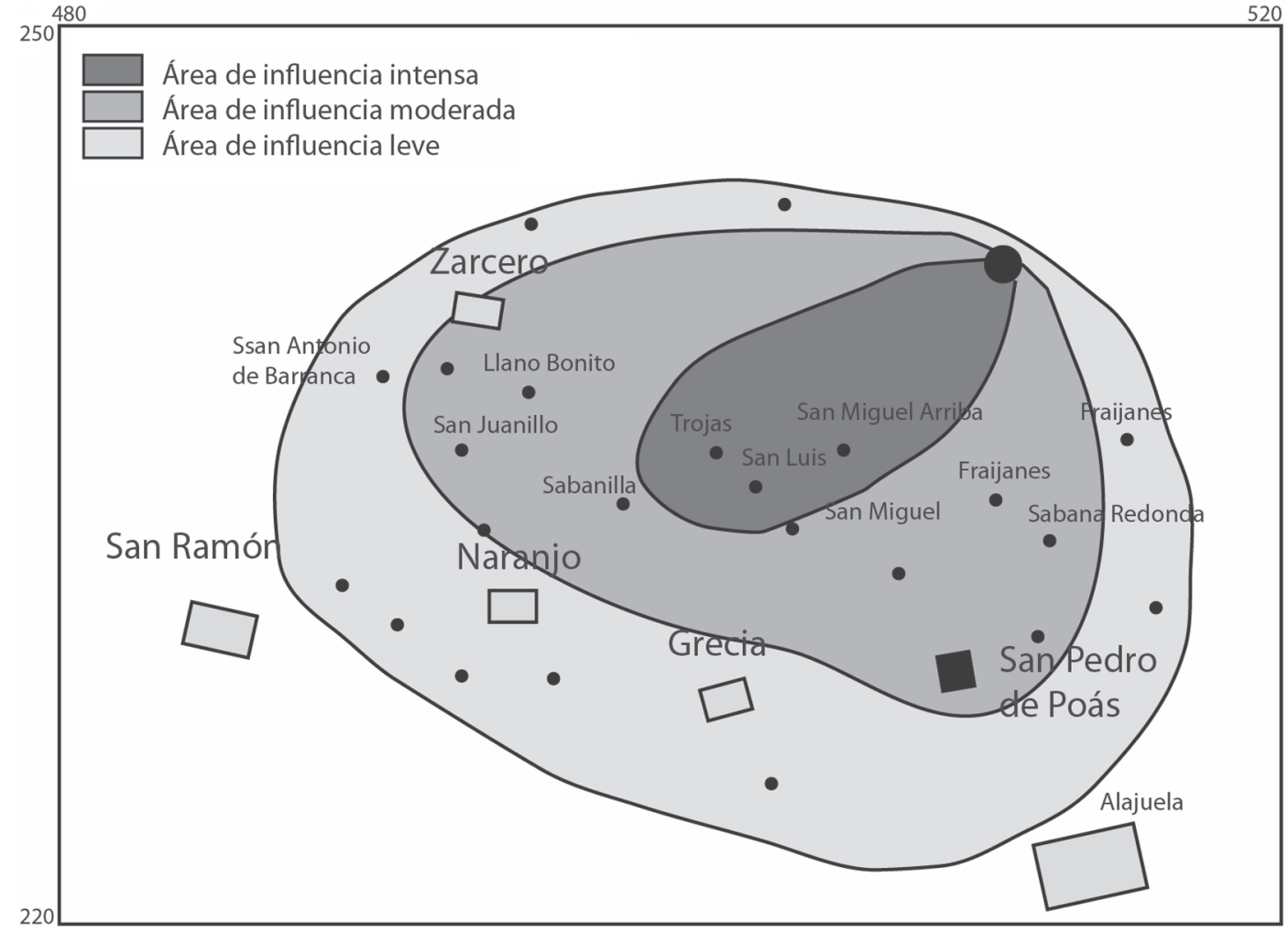

Fig. 2: Ubicación geográfica de los pueblos cercanos al volcán Poás (CNE, 1994).

alejándose del volcán. Sin embargo, algunos comentan que en caso de presentarse una erupción, preferirían trasladarse a las viviendas de otros familiares o amigos en lugar de evacuar completamente. Estos individuos dan mayor importancia a rodearse de sus seres queridos que a alejarse de las zonas de peligro. Algunos de los entrevistados tienen ideas erróneas sobre los lugares por donde evacuar ya que al no conocer con profundidad los peligros del volcán, no se dan cuenta que algunas zonas pueden ser afectadas por mayor cantidad de ceniza o lluvia ácida que otras.

Finalmente, se buscaba verificar qué tanto conoce los entrevistados sobre la existencia de planes de emergencia para el volcán Poás y si están al tanto de quienes son los miembros de algún comité de emergencias que pudieran servirles de ayuda en caso de presentarse una emergencia. A pesar de que un $40 \%$ de la población conocía sobre los planes de emergencias, nunca los han leído sino que simplemente han escuchado sobre su existencia, esta información les ha llegado por medio de sus hijos a quienes se les dan algunas instrucciones en las escuelas o colegios o porque han escuchado que existen planes para otros volcanes o sitios peligrosos y suponen que debe existir alguno para el volcán Poás.

En general, es posible analizar la percepción del riesgo de la población que vive en las zonas aledañas al volcán Poás con la encuesta realizada. Las personas que viven cerca del volcán Poás, han llegado a acostumbrarse y beneficiarse de las riquezas económicas que ofrece el área (agricultura de alta calidad y turismo), y suponen que no les va a pasar nada ya que han vivido toda su vida allí sin que ocurra una erupción de gran magnitud.

Esta idea de no ser personas que viven en una zona de riesgo, es lo que les ha impedido integrarse en el desarrollo de planes de emergencia ya sea a nivel local o comunal y que solo los habitantes que se encuentran en edad escolar sean los que 
conozcan un poco más sobre los peligros de vivir en una zona volcánica y sepan a quién acudir en caso de necesitar ayuda.

\section{PLAN DE EMERGENCIAS DEL VOLCÁN POÁS}

Los planes de emergencia definen las funciones, responsabilidades y procedimientos generales de reacción y alerta institucional, inventario de recursos, coordinación de actividades operativas y simulación para la capacitación y revisión, con el fin de salvaguardar la vida, proteger los bienes y recobrar la normalidad de la sociedad tan pronto como sea posible después de que se presente un fenómeno peligroso (UNESCO 1987).

Cuando se desea realizar un plan de emergencias volcánico, se asume que la comunidad expuesta al peligro volcánico tiene conciencia del riesgo para la vida y los bienes, y el deseo de una participación colectiva para reducirlo. Estos factores, tales como conciencia del riesgo y disposición a participar en iniciativas de prevención y mitigación se pueden medir a través de encuestas como las realizadas en este trabajo. Otros indicios a considerar son la distribución misma de la población, construcción de nuevas viviendas, entre otros, y el comportamiento de la población, apego a las regulaciones existentes, información de la comunidad, etc. El plan de emergencias requiere implementar un algún sistema de alerta para las erupciones, las cuales no son completamente predecibles y ocurren de forma inminente. Son precisamente esas características de las erupciones volcánicas que representan el mayor reto para que la población acate, entienda y justifique el plan de emergencias. La siguiente erupción podría ocurrir en días o cientos de anos. Dicha incertidumbre debe manejarse con cautela y desconfianza. Por ejemplo, se debe educar a la población de que existe una amenaza de erupción volcánica por medio de signos visibles de actividad volcánica. También, deben existir estaciones confiables de monitoreo científico de los volcanes, y finalmente mecanismos de alarma que se activen con el tiempo suficiente para permitir que se ejecuten las acciones apropiadas.
Para el período 2005-2006 solo existían 2 planes de Emergencia realizados para el volcán Poás. El primero de ellos es el Plan Operativo de Evacuación creado en 1994; en este plan se delimitan procesos y tareas de evacuación de la población, se describen los comités involucrados y cuáles son las tareas de cada una de las instituciones. Este plan solo se hace vigente cuando se declara Alerta Roja en el área. Seguidamente en 1999 se crea el Plan de Contingencia para Emergencias por Actividad del Volcán Poás. En este plan se agregan aspectos básicos no presentes en el plan de 1994, como por ejemplo una descripción general del volcán y los efectos que una erupción volcánica puede tener sobre la población.

Durante el 2006, la autora, en su tesis de maestría Escuela de Geología de la Universidad de Costa Rica, plantea una propuesta de plan de emergencia en el cual se agregan aspectos fundamentales no presentes en los planes anteriores. El plan de emergencia propuesto incluye en resumen los siguientes apartados:

1. Instancias involucradas en la atención de la emergencia: Comisión Nacional de Prevención de Riesgos y Atención de Emergencias, Comités Locales de Emergencia, Comités Comunales de Emergencia, Área de Conservación Cordillera Volcánica Central, Funcionarios del Parque Nacional Volcán Poás, Cruz Roja Costarricense, Ministerio de Salud, Cuerpo de Bomberos de Costa Rica, Dirección General de Tránsito, Fuerza Pública, Instituto Costarricense de Acueductos y Alcantarillados, Ministerio de Obras Públicas y Transporte, Red Sismológica Nacional (ICE UCR), Observatorio Vulcanológico y Sismológico de Costa Rica y la población civil.

2. Generalidades del Volcán Poás: Recuento de la geografía y geología del volcán.

3. Identificación de las amenazas por erupción volcánica.

4. Identificación y cartografía de las zonas amenazadas.

5. Censo de población: tomado del Censo de Costa Rica 2000.

6. Identificación de las alertas: referencia a los diferentes tipos de alerta: verde, amarilla, naranja y roja. 
7. Formulación y comunicación al público de las alertas a través de medios de comunicación, por ejemplo, anuncios en radio y televisión, y repartición de folletos, entre otros.

8. Identificación de las rutas de evacuación: La evacuación de la población se debe realizar cuando la Comisión Nacional de Prevención de Riesgos y Atención de Emergencias declaré alerta roja en la zona.

9. Identificación de puntos de reunión para personas en espera a ser evacuadas: Las personas que van a ser evacuadas deben ser organizadas por sus respectivos Comités Comunales de Emergencia y reubicadas en los salones comunales de su localidad, o en su defecto frente a la Iglesia principal del pueblo.

10. Medios de transporte, control de tráfico: Dirigido por la Dirección General de Tránsito.

11. Alojamiento y facilidades en las zonas de refugio: Las personas evacuadas tienen la posibilidad de hacer uso de los albergues temporales instalados en distintos poblados ubicados fuera del área de influencia o, por el contrario, pueden acudir a casas de familiares o amigos que se encuentren fuera de las áreas de influencia.

12. Inventario del personal y equipo para misiones de búsqueda y rescate: Coordinado por la Comisión Nacional de Prevención de Riesgos y Atención de Emergencias (CNE).

13. Hospitales y servicios médicos: El apartado de servicios médicos es responsabilidad de dos instituciones del país, particularmente la Cruz Roja Costarricense y el Ministerio de Salud.

14. Vigilancia de las zonas afectadas ante posibles amenazas secundarias: El Cuerpo de Bomberos de Costa Rica es el encargado de realizar las labores de vigilancia y prevención de posibles incendios en las zonas de afectación de la actividad volcánica.

15. Seguridad en las áreas evacuadas: La Fuerza Pública debe asumir las labores de seguridad y protección de las poblaciones a ser evacuadas.

16. Readecuación.

Cada uno de estos apartados deben ser analizados cada cierto tiempo de modo de que el plan de emergencias se mantenga actualizado y pueda ser aplicado ante una crisis volcánica.
Esta propuesta no se ha implementado formalmente hasta la fecha (Octubre 2010).

En resumen, la diferencia entre el plan de 1994 y 1999 es que el plan de 1999 incorpora la descripción general del volcán y los efectos que una erupción volcánica puede tener sobre la población. La propuesta del 2006 se diferencia de los planes existentes de 1994 y 1999 al integrar a la población afectada por medio de la incorporación de datos demográficos como número de habitantes (censo), mejoramiento de la comunicación del plan de emergencias a la comunidad a través de repartición de folletos describiendo el plan, etc. Además, se propone actualizar los mapas de evacuación con nuevas rutas específicas ya que los planes de 1994 y 1999 describen punto de salida y llegada pero no optimas rutas evacuación. Finalmente, quizás la diferencia más importante es que la propuesta del 2006 es la más actual, y por lo tanto, la que mejor se adecúa a las características actuales de la localidad. Además, dicho plan incluye un mecanismo de actualización y revisión periódica para incorporar nuevos datos, por ejemplo, futuros censos, creación de vías alternas, etc.

\section{CONCLUSIONES}

A lo largo del período de estudio de este trabajo (2005-2006) se pudo constatar que la población que vive en los alrededores del volcán Poás no ven al volcán como una amenaza para sus vidas y su economía, sino todo lo contrario, ellos se encuentran acostumbrados a vivir en sus laderas $\mathrm{y}$ aprovechan sus tierras fértiles para la agricultura y la ganadería. La encuesta realizada demostró que la mayoría de las personas que tienen mayores conocimientos sobre como actuar ante una posible emergencia debido a una erupción volcánica son aquellas en edad escolar o universitaria, a los cuales se les ha hablado de la problemática y han asistido a charlas sobre los riesgos de vivir en los alrededores del volcán Poás.

Por otra parte, es necesario dar a conocer los planes de emergencias y otros boletines informativos que se realicen con respecto a esta temática, 
ya que no basta con la instrucción que se les pueda dar a los niños en las escuelas y colegios. También resulta importante el informar a todos los sectores de la población, para evitar convertir la emergencia en un desastre mucho mayor.

Se propuso un Plan de Emergencia ante una actividad eruptiva del volcán Poás. Dicho plan muestra aspectos fundamentales que todo plan debe tener y propone algunos aspectos como por ejemplo las diferentes tipos de alertas, y las responsabilidades de cada institución según su área de trabajo.

Se debe actualizar el Plan de Emergencia del volcán Poás por lo menos cada cuatro años. Lo ideal sería actualizarlo de forma anual, de modo que siempre contenga la información más reciente y precisa al momento de ser aplicado. Por otra parte, se recomienda agregar otros aspectos en el apartado del área de salud, ya que este aspecto no se encuentra totalmente desarrollado dentro del Plan de Emergencia que se plantea en este trabajo.

Como recomendación para las instituciones encargadas de la atención de emergencias, es necesario dotar a los funcionarios que laboran dentro del Parque Nacional Volcán Poás, de materiales informativos para que estos puedan distribuirlos a los visitantes del área.

\section{AGRADECIMIENTOS}

A la Universidad de Costa Rica y en particular al grupo de profesores de la Maestría en Geología. Al Dr. Guillermo Alvarado por su gran aporte en este trabajo de investigación. Al Instituto Costarricense de Electricidad, La Comisión
Nacional de Prevención de Riesgos y Atención de Emergencias y al Área de Conservación Cordillera Volcánica Central por la información brindada, así como también a Elena Badilla, Raúl Mora, Lidier Esquivel y Carlos Espinoza por sus comentarios que mejoraron el presente trabajo.

\section{REFERENCIAS}

ALVARADO, G.E., 2000: Volcanes de Costa Rica.- 269 págs. Ed. Universidad Estatal a Distancia, San José.

BLUNDA, Y., 2006: Actualización del Plan de Emergencias del volcán Poás y el uso de la sismología volcánica como una herramienta preventiva.- 144 págs. Universidad de Costa Rica. [Tesis M.Sc.]

CASERTANO, L., BORGIA, A. \& CIGOLINI, C., 1983: El volcán Poás, Costa Rica: Cronología y características de la actividad.- Geof. Internacional, 22: 215-236

CNE, 1994: Plan operativo de evacuación zona de influencia del volcán Poás.- 26 págs, Comisión Nacional de Emergencias, San José. [Inf. Interno]

IBAÑEZ, J., 1995: Sismología Volcánica.51 págs. Teide European Laboratory Volcano, Madrid.

UNESCO, 1987: Manejo de emergencias volcánicas.- 82 págs, UNDRO UNESCO, New York. 


\section{APÉNDICE 1: \\ ENCUESTA APLICADA A POBLACIONES CERCANAS AL VOLCÁN POÁS}

1. Edad del entrevistado

$10-20 \_21-30 \_31-40 \_41-50 \_51-60 \_+60$

2. Motivos para vivir en la zona

Trabajo

Familia

Gusto

Otra

NR

3. ¿Considera usted que vive en una zona de peligro volcánico?

SI

$\mathrm{NO}$

NR

4. Usted estaría dispuesto a dejar sus propiedades en caso de presentarse una erupción volcánica

5. ¿Cuáles sitios considera usted los más peligrosos en esta área?

6. Sabría por qué vías evacuar en caso de una erupción volcánica? ¿Cuáles?

SI $\quad \mathrm{NO} \longrightarrow \mathrm{NR}$

7. ¿Conoce usted la existencia de Planes de Emergencia para el Volcán Poás?

$\mathrm{SI}$

8. ¿Conoce usted a los miembros del comité local de emergencia que lo pueden ayudar en caso de una erupción volcánica?

SI $\quad \mathrm{NO}$

9. ¿Cómo actuaría ante una erupción del volcán Poás? 